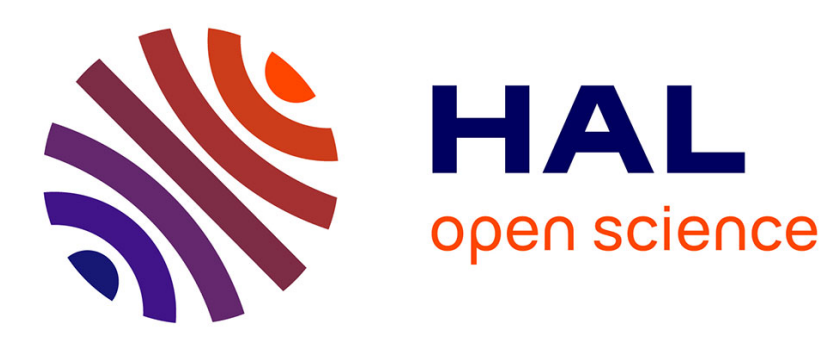

\title{
Semi-two-dimensional partitioning for parallel sparse matrix-vector multiplication
}

Enver Kayaaslan, Bora Uçar, Cevdet Aykanat

\section{To cite this version:}

Enver Kayaaslan, Bora Uçar, Cevdet Aykanat. Semi-two-dimensional partitioning for parallel sparse matrix-vector multiplication. PCO2015 (IPDPSW), May 2015, Hyderabad, India. pp.1125-1134. hal-01159692

\section{HAL Id: hal-01159692 \\ https://hal.inria.fr/hal-01159692}

Submitted on 3 Jun 2015

HAL is a multi-disciplinary open access archive for the deposit and dissemination of scientific research documents, whether they are published or not. The documents may come from teaching and research institutions in France or abroad, or from public or private research centers.
L'archive ouverte pluridisciplinaire HAL, est destinée au dépôt et à la diffusion de documents scientifiques de niveau recherche, publiés ou non, émanant des établissements d'enseignement et de recherche français ou étrangers, des laboratoires publics ou privés. 


\section{Semi-two-dimensional partitioning for parallel sparse matrix-vector multiplication}

\author{
Enver Kayaaslan, Bora Uçar \\ \{INRIA, CNRS $\}$ and LIP (UMR 5668: ENS Lyon, \\ CNRS, UCBL, Université de Lyon, INRIA), \\ 46 allee d'Italie, 69364, Lyon, France \\ Email: \{enver.kayaaslan, bora.ucar\}@ens-lyon.fr
}

\author{
Cevdet Aykanat \\ Bilkent University \\ 06800, Bilkent, Ankara, Turkey \\ Email: aykanat@cs.bilkent.edu.tr
}

\begin{abstract}
We propose a novel sparse matrix partitioning scheme, called semi-two-dimensional (s2D), for efficient parallelization of sparse matrix-vector multiply (SpMV) operations on distributed memory systems. In s2D, matrix nonzeros are more flexibly distributed among processors than one dimensional (rowwise or columnwise) partitioning schemes. Yet, there is a constraint which renders s2D less flexible than two-dimensional (nonzero based) partitioning schemes. The constraint is enforced to confine all communication operations in a single phase, as in 1D partition, in a parallel SpMV operation. In a positive view, s2D thus can be seen as being close to $2 \mathrm{D}$ partitions in terms of flexibility, and being close 1D partitions in terms of computation/communication organization. We describe two methods that take partitions on the input and output vectors of SpMV and produce S2D partitions while reducing the total communication volume. The first method obtains optimal total communication volume, while the second one heuristically reduces this quantity and takes computational load balance into account. We demonstrate that the proposed partitioning method improves the performance of parallel SpMV operations both in theory and practice with respect to $1 \mathrm{D}$ and $2 \mathrm{D}$ partitionings.
\end{abstract}

Keywords-Sparse matrix-vector multiplication, matrix partitioning.

\section{INTRODUCTION}

We consider efficient parallelization of sparse matrix-vector multiply (SpMV) operations of the form $\mathbf{y} \leftarrow \mathbf{A x}$ in distributed memory systems. Such a parallelization requires the data (the nonzeros of $\mathbf{A}$, the entries of the input vector $\mathbf{x}$ and the entries of the output vector $\mathbf{y}$ ) to be partitioned among available processors. According to the distribution of matrix elements, the data partitions are called one-dimensional (1D), or two-dimensional (2D). In 1D partitions, the matrix is partitioned rowwise or columnwise. In $2 \mathrm{D}$ partitions, the nonzeros in a row or in a column can be assigned to more than one processor. With 1D partitions, the parallel SpMV algorithms have a single communication phase in which either $\mathbf{x}$ - or $\mathbf{y}$-vector entries are communicated; whereas with 2D partitions, the parallel SpMV algorithms have two communication phases-first one is on $\mathbf{x}$ and the second one is on $\mathbf{y}$. In general, 2D partitions have more flexibility to be exploited in optimizing the parallel performance. On the other hand, 1D partitions has the advantage of confining the communication to a single phase. For a fair treatment of parallel SpMV, the associated matrix partitioning methods and a survey of methods the reader is referred to elsewhere [1, Chapter 4][5]. In this work, we propose a modification of the 2D-partitionbased parallel SpMV algorithm, where the communications on $\mathbf{x}$ and $\mathbf{y}$ are handled in a single phase as in the 1D-partitionbased parallel SpMV algorithm. This modification necessitates a special, 2D distribution of the nonzeros of the input matrix. We describe the characteristics of the required distribution and propose efficient heuristics to obtain them.

\section{A. Context}

Consider the fine-level computations $y_{i} \leftarrow y_{i}+a_{i j} \times x_{j}$ that compose $\mathbf{y} \leftarrow \mathbf{A x}$. The multiplication here is performed by the processor which holds the nonzero $a_{i j}$. The addition is performed by the same processor locally, and then it is possibly sent to the processor which is responsible to accumulate results on $y_{i}$. The standard SpMV algorithm based on such a fine-grain partition therefore proceeds in three phases:

1) (Expand) Each processor sends its input-vector entries $x_{j}$ to other processors that keep a nonzero $a_{i j}$.

2) Each processor performs the fine-multiplications for each $a_{i j}$ kept by the processor and accumulates to its own partial-result $\bar{y}_{i}$, that is, $\bar{y}_{i} \leftarrow \bar{y}_{i}+a_{i j} \times x_{j}$.

3) (Fold) Each processor receives the partial-results $\bar{y}_{i}$ from other processors that keep a nonzero $a_{i j}$ and aggregates them to obtain its output-vector entries $y_{i}$.

Step 1 Expand and Step 3 Fold are communication phases. When data partition is $1 \mathrm{D}$, one of these communication phases vanishes; e.g., in the columnwise partitioning, $a_{i j}$ and $x_{j}$ are kept together by the same processor, for each $a_{i j}$, and there is no communication in the expand phase. The 1D partitionings lead to reasonable performance in the parallel SpMV when the input matrix possesses an approximately regular number of nonzeros per row/or column. However, this is often not the case, and the parallel performance deteriorates due to poor computational balance and excessive use of bandwidth and latency. The 2D partitions come to rescue in these cases by not restricting nonzero partition on a column or row; but the parallel SpMV algorithm has both the expand and the fold phases and, hence, possibly increased number of messages.

Let us take a close look at the fine-level computations performed in the parallel SpMV, while assuming a data distribution. We can divide them into four groups: (i) both 
$x_{j}$ and $y_{i}$ are local; (ii) $x_{j}$ is local, $y_{i}$ is non-local; (iii) $y_{i}$ is local, $x_{j}$ is non-local; (iv) both $x_{j}$ and $y_{i}$ are non-local. Here, $x_{j}$ (similarly $y_{i}$ ) is called "local", if it is kept by the same processor that holds $a_{i j}$, and "non-local" otherwise. First, we note that the computational group (i) can be performed locally with no communication. Second, the group (ii) is independent of the expand phase, as $x_{j}$ entries are already present at the time of computation. However, the computations in (ii) should be carried out before the fold phase. Third, the computational group (iii) is suspended until the processor receives $x_{j}$ entries, yet the partial-results are not to be communicated since $y_{i}$ entries are local. The computational group (iv) requires special attention. Since both $x_{j}$ and $y_{i}$ entries are non-local, the computation is linked with both of the communication phases. The fine-multiplication with nonzeros in this group is the only reason that the expand phase has to precede the fold phase. Therefore, we can perform the two communication phases concurrently if the computational group (iv) has no nonzeros.

\section{B. Contributions and organization}

We propose semi-two-dimensional (s2D) data partitioning, which restricts each nonzero $a_{i j}$ to be together with either $x_{j}$ or $y_{i}$. By this restriction, we guarantee that the computational group (iv) is empty, and thus, the two communication phases can be fused into a single one. The s2D partitioning has more flexibility on the distribution of data than that of $1 \mathrm{D}$ methods, and requires single communication phase in parallel SpMV. This necessitates a reorganization in the parallel SpMV algorithm and new partitioning methods which are presented in Sections III and IV. Some recent work, summarized in Section $\mathrm{V}$, recognizes the inadequacy of $1 \mathrm{D}$ methods in modern applications and develop related alternatives. We perform some comparisons with them and present results in Section VI.

\section{BACKGROUND}

We collect material that we use later. Throughout the paper $K$ denotes the number of processors; data is partitioned into $K$ parts, each of which corresponds to a processor.

\section{A. $1 D$ (rowwise) partitioning}

In the $1 \mathrm{D}$ rowwise partitioning scheme, the sparse matrix $\mathbf{A}$ is partitioned in compliance with the output-vector y. That is, each processor $P_{k}$ holds an output-subvector $\mathbf{y}^{(k)}$ and a submatrix $\mathbf{A}^{(k)}$ such that $a_{i j} \in \mathbf{A}^{(k)}$ only if $y_{i} \in \mathbf{y}^{(k)}$. Thus, we can permute and write $\mathbf{A}$ as $\mathbf{A}=$ $\left[\mathbf{A}_{1 *}^{T} ; \mathbf{A}_{2 *}^{T} ; \ldots ; \mathbf{A}_{K *}^{T}\right]^{T}$. Processor $P_{k}$ holds the row-stripe $\mathbf{A}^{(k)}=\mathbf{A}_{k *}=\left[\mathbf{A}_{k 1}, \mathbf{A}_{k 2}, \ldots, \mathbf{A}_{k K}\right]$ and is responsible to compute $\mathbf{y}^{(k)} \leftarrow \mathbf{A}^{(k)} \mathbf{x}$. The input-vector $\mathbf{x}$ can be partitioned arbitrarily. Then, the input- and output-vector partition induces the block structure on $\mathbf{A}$ such that $a_{i j} \in \mathbf{A}_{\ell k}$ only if $y_{i} \in \mathbf{y}^{(\ell)}$ and $x_{j} \in \mathbf{x}^{(k)}$.

\section{B. The Dulmage-Mendelsohn decomposition}

Let $\mathbf{B}$ be an arbitrary rectangular matrix, and $\hat{m}(\mathbf{B})$ and $\hat{n}(\mathbf{B})$ represent the number of nonempty rows and the number of nonempty columns in $\mathbf{B}$, respectively. Then, we build the Dulmage-Mendelsohn (DM) decomposition [10] on B as,

$$
\hat{\mathbf{B}}=\left[\begin{array}{ccc}
\mathbf{H} & \mathrm{X} & \mathrm{Z} \\
0 & \mathbf{S} & \mathrm{Y} \\
0 & 0 & \mathbf{V}
\end{array}\right] \quad \mathbf{B}=\left[\begin{array}{cc}
\hat{\mathbf{B}} & 0 \\
0 & 0
\end{array}\right]
$$

where $\hat{\mathbf{B}}$ is the nonzero block of $\mathbf{B}$. Here $\mathbf{H}, \mathbf{S}$, and $\mathbf{V}$ refer to horizontal, square, and vertical blocks of $\mathbf{B}$, respectively. As their names suggest, we have $\hat{m}(\mathbf{H})<\hat{n}(\mathbf{H}), \hat{m}(\mathbf{S})=\hat{n}(\mathbf{S})$, and $\hat{m}(\mathbf{V})>\hat{n}(\mathbf{V})$. This block triangular decomposition is based on the canonical decomposition of bipartite graphs induced by maximum matchings [10], [15]. This implies that $\hat{m}(\mathbf{H})+\hat{m}(\mathbf{S})+\hat{n}(\mathbf{V})$ is the minimum number of rows and columns required to cover all nonzeros.

\section{PARALLEL SPMV FOR S2D PARTITIONING}

In this section, we introduce $\mathrm{s} 2 \mathrm{D}$ partitioning. We also show how to modify the parallel SpMV in accordance with this partitioning to obtain a single phase of communication.

Let $\mathbf{A}$ be an $m \times n$ sparse matrix, and $\mathbf{x}$ and $\mathbf{y}$ be dense input- and output-vectors of size $n$ and $m$, respectively. In s2D partitioning, the matrix $\mathbf{A}$ and the vectors $\mathbf{x}$ and $\mathbf{y}$ are partitioned into $K$ parts, where each processor $P_{k}$ holds submatrix $\mathbf{A}^{(k)}$, and subvectors $\mathbf{x}^{(k)}$ and $\mathbf{y}^{(k)}$ such that $a_{i j} \in \mathbf{A}^{(k)}$ only if $x_{j} \in \mathbf{x}^{(k)}$ or $y_{i} \in \mathbf{y}^{(k)}$. Then, $\mathbf{A}$ can be permuted and written as

$$
\mathbf{A}=\mathbf{A}^{(1)}+\mathbf{A}^{(2)}+\cdots+\mathbf{A}^{(K)},
$$

where

$$
\mathbf{A}^{(k)}=\left[\begin{array}{cccccc} 
& & & \mathbf{A}_{1 k}^{(k)} & & \\
& & & \mathbf{A}_{2 k}^{(k)} & & \\
& & & \vdots & & \\
\mathbf{A}_{k 1}^{(k)} & \mathbf{A}_{k 2}^{(k)} & \cdots & \mathbf{A}_{k k} & \cdots & \mathbf{A}_{k K}^{(k)} \\
& & & \vdots & & \\
& & & \mathbf{A}_{K k}^{(k)} & &
\end{array}\right],
$$

or each $1 \leq k \leq K$. The off-diagonal block $\mathbf{A}_{\ell k}$ induced by $\mathbf{y}^{(\ell)}$ and $\mathbf{x}^{(k)}$, where $\ell \neq k$, can now be written as

$$
\mathbf{A}_{\ell k}=\mathbf{A}_{\ell k}^{(k)}+\mathbf{A}_{\ell k}^{(\ell)} \text {. }
$$

An s2D partition is therefore can be considered as a 2D partition where $\mathbf{A}_{q r}^{(k)} \neq \mathbf{0}$ only if $q=k$ or $r=k$.

In Section I, we presented a categorization of computational work into four groups. The key observation is that an s2D partition admits only the computational groups having either $x_{j}$ or $y_{i}$ local with respect to $a_{i j}$. One can fuse the expand and the fold communications in a single phase, if the task of computational group (ii) " $x_{j}$ is local, $y_{i}$ is non-local" is relocated prior to the expand phase. In the modified parallel SpMV, each processor $P_{k}$ executes the following steps:

1) (Precompute) For each $\ell \neq k$;

a) Compute $\mathbf{y}_{k}^{(\ell)} \leftarrow \mathbf{A}_{\ell k}^{(k)} \mathbf{x}^{(k)}$,

b) Form vector $\hat{\mathbf{y}}_{k}^{(\ell)}$, which contains only those entries of $\mathbf{y}_{k}^{(\ell)}$ corresponding to nonzero rows in $\mathbf{A}_{\ell k}^{(k)}$. 
2) (Expand-and-Fold) For each $\ell \neq k$;

a) Form vector $\hat{\mathbf{x}}_{\ell}^{(k)}$, which contains only those entries of $\mathbf{x}^{(k)}$ corresponding to nonzero columns in $\mathbf{A}_{\ell k}^{(\ell)}$,

b) Send vector $\left[\hat{\mathbf{x}}_{\ell}^{(k)}, \hat{\mathbf{y}}_{k}^{(\ell)}\right]$ to processor $P_{\ell}$.

3) (Compute) Compute the output-subvector $\mathbf{y}^{(k)}$ as

$$
\mathbf{y}^{(k)} \leftarrow \sum_{\ell \neq k} \mathbf{A}_{k \ell}^{(k)} \hat{\mathbf{x}}_{k}^{(\ell)}+\mathbf{A}_{k k} \mathbf{x}^{(k)}+\sum_{\ell \neq k} \hat{\mathbf{y}}_{\ell}^{(k)} .
$$

This modified parallel SpMV is composed of three phases, where two of them are computational. The first phase (Precompute) computes the partial-results associated with nonze$\operatorname{ros} a_{i j}$ such that $x_{j}$ is local and $y_{i}$ is non-local. The processor $P_{k}$ computes the partial-result vector $\hat{\mathbf{y}}_{k}^{(\ell)}=\left[\bar{y}_{i}: a_{i j} \in \mathbf{A}_{\ell k}^{(k)}\right]$ to be sent to processor $P_{\ell}$ for each $\ell \neq k$. The second phase (Expand-and-Fold) is the one that performs the communication. The partial-result vector $\hat{\mathbf{y}}_{k}^{(\ell)}$ is accompanied with the vector $\hat{\mathbf{x}}_{\ell}^{(k)}$ of input-vector entries required by $P_{\ell}$, that is $\hat{\mathbf{x}}_{\ell}^{(k)}=\left[\begin{array}{ll}x_{j}: a_{i j} \in \mathbf{A}_{\ell k}^{(\ell)}\end{array}\right]$. Then, $P_{k}$ sends the combined vector $\left[\hat{\mathbf{x}}_{\ell}^{(k)}, \hat{\mathbf{y}}_{k}^{(\ell)}\right]$ to $P_{\ell}$. In the third phase (Compute), the associated output-subvector $\mathbf{y}^{(k)}$ is computed by aggregating the partial-results of three kinds of sources: $\mathbf{A}_{k \ell}^{(k)}(\ell \neq k)$, $\mathbf{A}_{k k}$, and $\mathbf{A}_{k \ell}^{(\ell)}(\ell \neq k)$. The first one is the nonzero block $\mathbf{A}_{k \ell}^{(k)}$ where $\ell \neq k$, and $\hat{\mathbf{x}}_{k}^{(\ell)}$ refers to the input-subvector received during the Expand-and-Fold phase from processor $P_{\ell}$. Here $\hat{\mathbf{x}}_{k}^{(\ell)}$ is required to perform fine-multiplications associated with nonzeros in $\mathbf{A}_{k \ell}^{(k)}$. The second one is the diagonal block $\mathbf{A}_{k k}$, which is also expected to contain the bulk of computation for efficiency. The third source type is the nonzero block $\mathbf{A}_{k \ell}^{(\ell)}$ where $\ell \neq k$. The associated fine-multiplications have already been performed (in Precompute), and the partial results are received (in Expand-and-Fold). Thus, $P_{k}$ just accumulates those partial-results $\hat{\mathbf{y}}_{\ell}^{(k)}$ to the output-subvector $\mathbf{y}^{(k)}$.

We make three observations on this modified parallel SpMV. First, a message is communicated from $P_{k}$ to $P_{\ell}$ only when $\left[\hat{\mathbf{x}}_{\ell}^{(k)}, \hat{\mathbf{y}}_{k}^{(\ell)}\right]$, and equivalently $\mathbf{A}_{\ell k}$, is nonemtpy. Notice that the input- and output-vector partitions are the only factor that determines whether $\mathbf{A}_{\ell k}$ is empty or not. Therefore, the communication patterns for s2D and 1D partitions are identical whenever they have vector partitions in common. Second, $P_{k}$ sends the input-vector entries $\hat{\mathbf{x}}_{\ell}^{(k)}$ and the partialresults $\hat{\mathbf{y}}_{k}^{(\ell)}$ required by $P_{\ell}$ in a single message. Thus, s2D partitioning not only simplifies the parallel SpMV by coupling the two communication phases, but can also reduce the latency overhead with respect to $2 \mathrm{D}$ methods. The last observation is that the modification has no effect on being a generalization of row-parallel SpMV. That is, when the partition is 1D rowwise, the precompute phase is not needed, and the single communication boils down to the expand phase.

We now give the formulation for the communication volume (bandwidth) as done in similar studies [18]. $P_{k}$ sends an inputvector entry $x_{j} \in \mathbf{x}^{(k)}$ to $P_{\ell}$, whenever the corresponding column in $\mathbf{A}_{\ell k}^{(\ell)}$ has a nonzero. Thus, the communication volume $\lambda_{k \rightarrow \ell}^{\mathrm{x}}$ incurred by the input-vector entries is

$$
\lambda_{k \rightarrow \ell}^{\mathrm{x}}=\left|\hat{\mathbf{x}}_{\ell}^{(k)}\right|=\left|\left\{x_{j} \in \mathbf{x}^{(k)}: a_{i j} \in \mathbf{A}_{\ell k}^{(\ell)}\right\}\right|=\hat{n}\left(\mathbf{A}_{\ell k}^{(\ell)}\right) .
$$

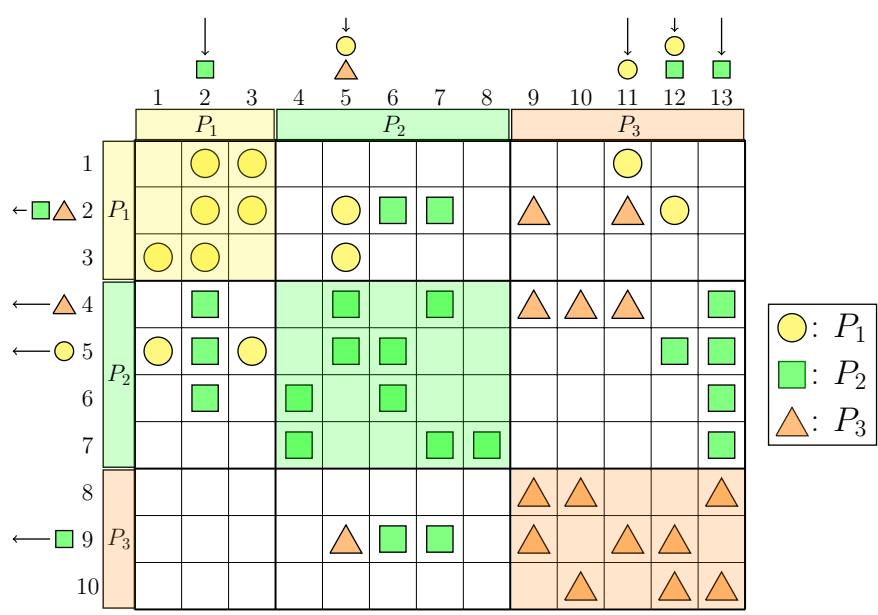

Fig. 1: A sample s2D partitioning of $10 \times 13$ sparse matrix. The shapes above the columns and left to the rows indicate communication requirements due to the corresponding column or row; e.g., $P_{2}$ sends $\left[x_{5}, \bar{y}_{2}\right]$ to $P_{1}$ in a single message, where input-vector entry $x_{5}$ is needed due to $a_{2,5}$ and partial result $\bar{y}_{2}$ is precomputed by $P_{2}$ as $\bar{y}_{2}=a_{2,6} \times x_{6}+a_{2,7} \times x_{7}$.

Likewise, $P_{\ell}$ receives a partial result $\bar{y}_{i} \in \hat{\mathbf{y}}_{k}^{(\ell)}$ from $P_{k}$, whenever the corresponding row in $\mathbf{A}_{\ell k}^{(k)}$ has a nonzero. Then, the communication volume $\lambda_{k \rightarrow \ell}^{\mathrm{y}}$ due to the partial results is

$$
\lambda_{k \rightarrow \ell}^{\mathrm{y}}=\left|\hat{\mathbf{y}}_{k}^{(\ell)}\right|=\left|\left\{y_{i} \in \mathbf{y}^{(\ell)}: a_{i j} \in \mathbf{A}_{\ell k}^{(k)}\right\}\right|=\hat{m}\left(\mathbf{A}_{\ell k}^{(k)}\right),
$$

and the total communication volume $\lambda_{k \rightarrow \ell}$ from $P_{k}$ to $P_{\ell}$ is

$$
\lambda_{k \rightarrow \ell}=\hat{n}\left(\mathbf{A}_{\ell k}^{(\ell)}\right)+\hat{m}\left(\mathbf{A}_{\ell k}^{(k)}\right) .
$$

Figure 1 displays a $10 \times 13$ sparse matrix $\mathbf{A}$ along with a sample 3-way s2D partitioning on it. Notice that each nonzero is assigned to either its row part or column part, e.g., $a_{2,5}$ is assigned to its row part $P_{1}$, whereas $a_{2,6}$ is assigned to its column part $P_{2}$. Also notice that nonzeros of diagonal blocks are assigned to their corresponding parts. $P_{1}$ requires $x_{5}$ from $P_{2}$ for fine-level multiplication with $a_{2,5}$ and $a_{3,5}$. On the other hand, the partial result $\bar{y}_{2}$ is also needed to be communicated from $P_{2}$ to $P_{1}$, where $P_{2}$ has precomputed $\bar{y}_{2}=a_{2,6} \times x_{6}+a_{2,7} \times x_{7}$. Therefore, in the Expand-andFold phase, $P_{2}$ sends $\left[x_{5}, \bar{y}_{2}\right]$ to $P_{1}$ within a single packet. In the figure, a circle, rectangle, or, triangle upon the columns indicate that the respective $\mathrm{x}$-vector entry is required for the corresponding part. For instance, $P_{2}$ is the only processor that requires $x_{13}$, since $P_{2}$ is the only one that has nonzeros in that column. Similarly, a shape left to rows indicate that the partial results of the respective $\mathbf{y}$-vector entry is communicated from the corresponding part, e.g., $P_{1}$ sends a partial result $\bar{y}_{5}$ to $P_{2}$ due to $a_{5,1}$ and $a_{5,3}$. Here, the total communication volume $\lambda_{3 \rightarrow 2}$ from $P_{3}$ to $P_{2}$ is 3 , as $\hat{n}\left(\mathbf{A}_{2,3}^{(2)}\right)=2$ and $\hat{m}\left(\mathbf{A}_{2,3}^{(3)}\right)=1$.

\section{A COMBINATORIAL METHOD FOR S2D PARTITIONING}

We propose an s2D partitioning method with two steps. The first step accepts an input- and output-vector partition into $K$ parts. In the second step, we build an s2D partition with respect to that vector partition. The choice of vector 
partitioning in the first step is related to how an s2D partition is built upon in the second step. In the proposed approach, 1D rowwise partitioning is the most relevant one to obtain a vector partition, since we initially assign nonzeros together with their corresponding output vector entries, and then apply a coarse-grain refinement.

We formalize s2D nonzero partitioning problem as follows.

Problem 1: Given a $K$-way partition on $\mathrm{x}$ and a $K$-way partition on $\mathbf{y}$, find a $K$-way partition on $\mathbf{A}$ such that for each nonzero $a_{i j}, \pi\left(a_{i j}\right) \in\left\{\pi\left(y_{i}\right), \pi\left(x_{j}\right)\right\}$, where $\pi(\cdot)$ refers to the owner of a data element.

Let each processor $P_{k}$ have an input-subvector $\mathbf{x}^{(k)}$ and an output-subvector $\mathbf{y}^{(k)}$. This vector partition induces a $K$ by $K$ block structure on $\mathbf{A}$, where

$$
\mathbf{A}_{\ell k}=\left\{a_{i j}: y_{i} \in \mathbf{y}^{(\ell)}, x_{j} \in \mathbf{x}^{(k)}\right\} .
$$

Our aim is to build two blocks $\mathbf{A}_{\ell k}^{(k)}$ and $\mathbf{A}_{\ell k}^{(\ell)}$ for each offdiagonal block $\mathbf{A}_{\ell k}$, which is induced by the input- and outputvector partition, such that

$$
\mathbf{A}_{\ell k}=\mathbf{A}_{\ell k}^{(k)}+\mathbf{A}_{\ell k}^{(\ell)} .
$$

We first show how to find such an s2D partition with optimal total communication volume

$$
\lambda=\sum_{\ell, k} \lambda_{k \rightarrow \ell},
$$

for the given input- and output-vector partition. Then, we take the load balance into account, and propose a heuristic to obtain an s2D partition that reduces both the maximum computational load and the total communication volume.

\section{A. Optimizing the total communication volume}

As the blocks are disjoint, the total communication volume $\lambda$ given in (4) can be minimized by optimizing the communication volume of each off-diagonal block independently. Below, we describe how to minimize the communication volume $\lambda_{k \rightarrow \ell}$ of an off-diagonal block $\mathbf{A}_{\ell k}$.

We first obtain the DM decomposition of $\mathbf{A}_{\ell k}$ as

$$
\widehat{\mathbf{A}}_{\ell k}=\left[\begin{array}{ccc}
\mathbf{H}_{\ell k} & \times & \times \\
0 & \mathbf{S}_{\ell k} & \times \\
0 & 0 & \mathbf{V}_{\ell k}
\end{array}\right], \quad \mathbf{A}_{\ell k}=\left[\begin{array}{cc}
\widehat{\mathbf{A}}_{\ell k} & 0 \\
0 & 0
\end{array}\right] \text {. }
$$

Based on this DM decomposition, we build two blocks $\mathbf{A}_{\ell k}^{(\ell)}$ and $\mathbf{A}_{\ell k}^{(k)}$ as

$$
\widehat{\mathbf{A}}_{\ell k}^{(\ell)}=\left[\begin{array}{cc}
\times & \times \\
\mathbf{S}_{\ell k} & \times \\
0 & \mathbf{V}_{\ell k}
\end{array}\right], \quad \widehat{\mathbf{A}}_{\ell k}^{(k)}=\mathbf{H}_{\ell k},
$$

where $\widehat{\mathbf{A}}_{\ell k}^{(\ell)}$ and $\widehat{\mathbf{A}}_{\ell k}^{(k)}$ refer to the nonzero block of $\mathbf{A}_{\ell k}^{(\ell)}$ and $\mathbf{A}_{\ell k}^{(k)}$, respectively. Due to the formula given in (3), we have

$$
\lambda_{k \rightarrow \ell}=\hat{n}\left(\mathbf{A}_{\ell k}^{(\ell)}\right)+\hat{m}\left(\mathbf{A}_{\ell k}^{(k)}\right) .
$$

The DM decomposition suggests that $\lambda_{k \rightarrow \ell}$, when computed as in (6), is the minimum that can be achieved by distributing nonzeros of $\mathbf{A}_{\ell k}$ into two blocks $\mathbf{A}_{\ell k}^{(k)}$ and $\mathbf{A}_{\ell k}^{(\ell)}$.

\section{B. Bi-objective optimization}

The computational load of a processor is proportional to the number of nonzeros assigned to it. Hence, for an s2D partition as in (1), the computational load of a given processor $P_{k}$ is

$$
\left|\mathbf{A}^{(k)}\right|=\sum_{\ell \neq k}\left|\mathbf{A}_{k \ell}^{(k)}\right|+\left|\mathbf{A}_{k k}\right|+\sum_{\ell \neq k}\left|\mathbf{A}_{\ell k}^{(k)}\right| .
$$

Then, we compute the maximum computational load $\widetilde{W}$ as

$$
\widetilde{W}=\max _{k}\left|\mathbf{A}^{(k)}\right| \text {. }
$$

Our aim is to minimize both the total communication volume $\lambda$ and the maximum computational load $\widetilde{W}$. As we need to handle two different objectives, we consider the following problem: Given an upper bound $W_{\lim }$ to the maximum computational load, find an s2D partition with respect to the given input- and output-vector partition such that $W \leq W_{\text {lim }}$ and $\lambda$ is minimum.

We follow a coarse-grain approach that adapts the one used in optimizing the total communication volume. We consider two alternatives for each off-diagonal block $\mathbf{A}_{\ell k}$ :

$$
\begin{aligned}
& \text { (A1) } \mathbf{A}_{\ell k}^{(\ell)}=\mathbf{A}_{\ell k} \text {, and } \mathbf{A}_{\ell k}^{(k)}=\mathbf{0} \text {, } \\
& \text { (A2) } \mathbf{A}_{\ell k}^{(\ell)}=\mathbf{A}_{\ell k}-\mathbf{H}_{\ell k} \text { and } \mathbf{A}_{\ell k}^{(k)}=\mathbf{H}_{\ell k} \text { (cf. (5)). }
\end{aligned}
$$

One obtains

$$
\begin{aligned}
\lambda_{k \rightarrow \ell}^{\mathrm{A} 1} & =\hat{n}\left(\mathbf{A}_{\ell k}\right), \\
\lambda_{k \rightarrow \ell}^{\mathrm{A} 2} & =\hat{n}\left(\mathbf{A}_{\ell k}-\mathbf{H}_{\ell k}\right)+\hat{m}\left(\mathbf{H}_{\ell k}\right),
\end{aligned}
$$

with the first and second alternatives, respectively. Recall that $\lambda_{k \rightarrow \ell}^{\mathrm{A} 2}$ is the minimum that can be achieved. Then, we reduce our problem to choose an alternative for each off-diagonal block so that $\lambda$ is minimum while $\widetilde{W} \leq W_{\text {lim }}$. This problem is NP-complete (contains Knapsack as a special case), and we resort to heuristics.

We propose Algorithm 1. In this algorithm, we start with choosing the alternative (A1) for all off-diagonal blocks. This initial partitioning coincides with the $1 \mathrm{D}$ rowwise partitioning of $\mathbf{A}$ with respect to given input and output vector partition. Then, we go over the off-diagonal blocks multiple times, to alternate the choices from (A1) to (A2). Any alternation is final-we do not alternate our choice back to (A1). The iterations over the off-diagonal blocks are in decreasing order of the associated reductions in the communication volume. This reduction, which is denoted by $\lambda_{\ell k}^{-}$, is equal to the number of columns minus the number of rows of the corresponding horizontal block. That is, for an off-diagonal block $\mathbf{A}_{\ell k}$,

$$
\lambda_{\ell k}^{-}=\lambda_{k \rightarrow \ell}^{\mathrm{A} 2}-\lambda_{k \rightarrow \ell}^{\mathrm{A} 1}=\hat{n}\left(\mathbf{H}_{\ell k}\right)-\hat{m}\left(\mathbf{H}_{\ell k}\right) .
$$

At the moment of deciding to alternate the choice of an offdiagonal block or not, our only consideration is the maximum computational load. That is, we check if the alternation cause the maximum computational load exceed the limit. Notice that this algorithm assumes the initial maximum computational load $\widetilde{W}=\max _{k}\left|\mathbf{A}_{k *}\right|$ is less than $W_{\text {lim. }}$. However, this may not necessarily be the case. Therefore, we implement the algorithm such that we check if $W_{k}+\left|\mathbf{H}_{\ell k}\right|$ is smaller than the current maximum load $\widetilde{W}$ instead of $W_{\text {lim }}$, whenever $\widetilde{W}>W_{\lim }$ during the execution of the algorithm. 


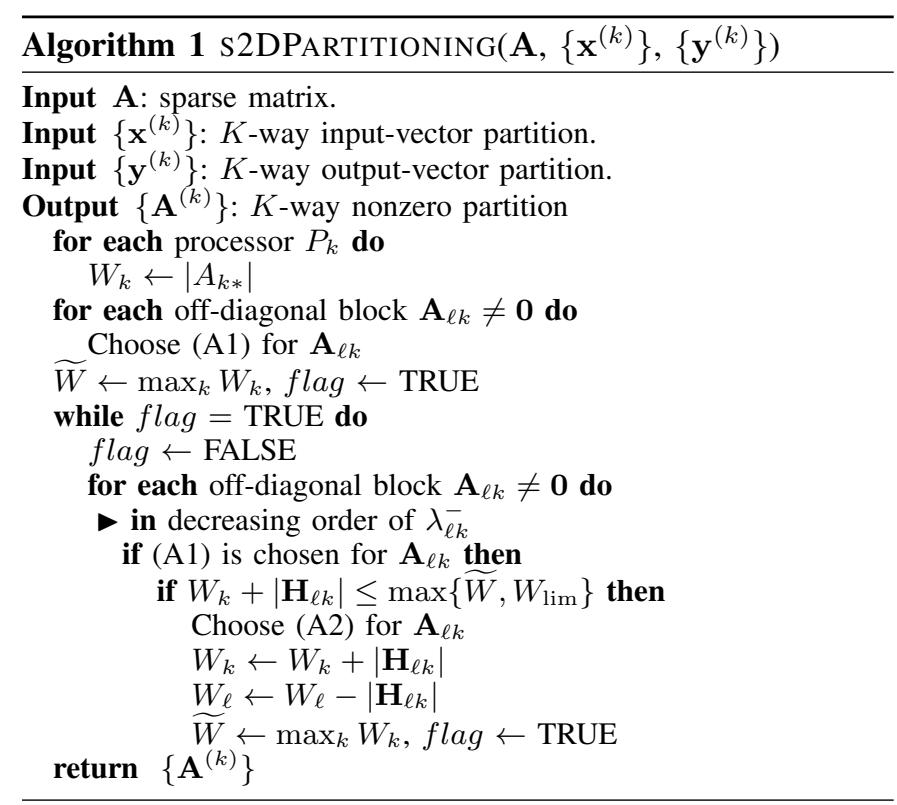

\section{RELATED RECENT WORK AND COMPARISON}

We summarize some recent work on partitioning methods for parallel SpMV in modern applications.

Kuhlemann and Vassilevski [12] recognize the need to reduce the number of messages in parallel SpMV operations with matrices corresponding to scale-free graphs. They present methods to embed the given graph in a bigger one to reduce the number of messages. The gist of the method is to split a vertex into a number of copies (the number is determined with a simple calculation to limit the maximum number of messages per processor). In such a setting, the SpMV operations with the matrix associated with the original graph, $\mathbf{y} \leftarrow \mathbf{A x}$, is then cast as triple sparse matrix vector products of the form $\mathbf{y} \leftarrow \mathbf{Q}^{T}(\mathbf{B}(\mathbf{Q x}))$, much like in multigrid solvers. This original work can be extended to other matrices (not necessarily symmetric, nor square) by recognizing the triplet product as a communication on $\mathbf{x}$ for duplication (for the columns that are split), communication of $\mathbf{x}$ vector entries (duplicates are associated with different destinations), multiplication, and as a communication on the output vector (for the rows that are split) to gather results. This exciting extension requires further analysis, which is out of our scope in this paper.

Boman et al. [2] propose a 2D partitioning method obtained by post-processing a 1D partition. Given a 1D partition among $P$ processors, the method maps the $P \times P$ block structure to a virtual mesh of size $P_{r} \times P_{c}$ and reassigns the off-diagonal blocks so as to limit the number of messages per processor by $P_{r}+P_{c}$. The post-processing is fast, and hence the method is as nearly efficient as a $1 \mathrm{D}$ partitioning method. However, the communication volume and the load balance obtained in the 1D partitioning phase are disturbed (this does not seem to cause problems for their applications), and the method does not have any means to control the perturbation.

Pelt and Bisseling [14] propose a model to partition sparse matrices into two parts (which then can be used recursively to partition into any number of parts). The essential idea has two steps. First, the nonzeros of a given matrix $\mathbf{A}$ are split into two different matrices (of the same size as the original matrix), say $\mathbf{A}=\mathbf{A}_{r}+\mathbf{A}_{c}$. Second, $\mathbf{A}_{r}$ and $\mathbf{A}_{c}$ are partitioned together, where $\mathbf{A}_{r}$ is partitioned rowwise, and $\mathbf{A}_{c}$ is partitioned columnwise. As all nonzeros of $\mathbf{A}$ are in only one of $\mathbf{A}_{r}$ or $\mathbf{A}_{c}$, the final result is a two-way partitioning of the nonzeros of $\mathbf{A}$. The resulting partition on $\mathbf{A}$ achieves load balance and reduces the total communication volume by the standard hypergraph partitioning techniques. Consider the following interpretation of this approach. First split the matrix as before $\mathbf{A}=\mathbf{A}_{r}+\mathbf{A}_{c}$. Then, build a composite hypergraph model [17] using the enriched column-net hypergraph for $\mathbf{A}_{r}$ and the row-net hypergraph for $\mathbf{A}_{c}$ and amalgamate the vertices as required. Now consider a $K$-way partition of the composite model. In this partition, a column in $\mathbf{A}_{c}$ is assigned to a part which holds the corresponding $\mathbf{x}$ vector entry. Similarly, a row in $\mathbf{A}_{r}$ is assigned to a part which holds the corresponding $\mathbf{y}$ vector entry. Therefore, the output from this partition can be decoded as an s2D partition. A few notes are necessary. First, in a preliminary set of experiments we tested the use of composite hypergraph models and performed a standard hypergraph partitioning on it (instead of using splits and iterative refinement at every recursive bisection step as done by Pelt and Bisseling). Our tests agreed with the reported results [14] in that the medium-grain approach improved upon the fine-grain [6] method. Second, the use of composite models enable obtaining symmetric vector partitions (that is the partitions on $\mathbf{x}$ and $\mathbf{y}$ are the same), while exactly encoding the total communication volume. To the best of our understanding, a symmetric vector partitioning is not always possible with the original approach of Pelt and Bisseling using the standard hypergraph partitioning methods.

Two-dimensional partitioning methods that bound the maximum number of messages per processor, such as the checkerboard [5], [7] and orthogonal recursive bisection [18] based, have been used in modern applications [19], [21], sometimes without graph/hypergraph partitioning [20]. In almost all cases, inadequacy of 1D partitioning schemes are confirmed.

All these previous work assumes the standard SpMV multiplication algorithm based on expanding $\mathrm{x}$-vector entries, performing multiplies with matrix entries, and folding $\mathbf{y}$-vector entries. Compared to all these previous work, ours has therefore a distinctive characteristic. We search for a special 2D partition on the matrix elements in which a nonzero is assigned to a processor holding the associated input or output vector entry. This is essential for the proposed SpMV algorithm in which the expand and fold messages are combined. The implication is that there is a single communication phase (all the previous work based on 2D partitions has two communication phase) as is the case when 1D partitions are used; yet there is also a greater flexibility to reduce the communication volume than the $1 \mathrm{D}$ method. In the next section, we compare the proposed method with the well-known 1D and 2D partitioning schemes, together with the schemes proposed by Boman et al. [2] and Pelt and Bisseling [14]. 


\begin{tabular}{l|rrrr|l} 
name & $n$ & $n n z$ & $d_{\text {avg }}$ & $d_{\max }$ & application \\
\hline crystk02 & 13965 & 968583 & 69.4 & 81 & materials problem \\
turon_m & 189924 & 1690876 & 8.9 & 11 & structural engineering \\
trdheim & 22098 & 1935324 & 87.6 & 150 & structural engineering \\
c-big & 345241 & 2340859 & 6.8 & 19578 & non-linear optimization \\
ASIC_680k & 682862 & 2638997 & 3.9 & 388488 & circuit simulation \\
3dtube & 45330 & 3213618 & 70.9 & 2364 & structural engineering \\
pkustk12 & 94653 & 7512317 & 79.4 & 4146 & structural engineering \\
pattern1 & 19242 & 9323432 & 484.5 & 6028 & optimization problem \\
\hline
\end{tabular}

TABLE I: Properties of the test matrices used in comparison with 1D and 2D methods. The sparse matrices are displayed in increasing order of their nonzero counts $(n n z)$. Here, $d_{\text {avg }}$ and $d_{\max }$ are the average and the maximum number of nonzeros of rows, respectively.

\section{EXPERIMENTAL RESULTS}

We evaluate the performance of the s2D partitioning (s2D) in two setups. In the first one (results are in Section VI-A), we compare it with the 1D rowwise [3] (1D) and the 2D finegrain [6] (2D) partitioning methods with $K \in\{16,64,256\}$ processors on a set of eight sparse matrices from the University of Florida Matrix (UFL) Collection [9]. We also measure SpMV timings on a Cray XE6 system, and we observe that $\mathrm{s} 2 \mathrm{D}$ displays considerable improvement on the overall execution times with respect to both $1 \mathrm{D}$ and $2 \mathrm{D}$, on average. However, as also observed in related studies (e.g., [11], [16]), the latency overhead dominates when $K$ gets larger. Therefore, we build the second setup, which mainly focuses on the partitioning methods that bound latency overhead of a processor. For the second setup (results are in Section VI-B), we consider another set of eight sparse matrices, which contain high degree rows, from UFL Collection [9] and Stanford Network Analysis Platform (SNAP) [13]. These matrices naturally cause very high number of messages sent by a processor, and thus parallel computation with bounded latency is required. We measure the performance results with $K \in\{256,1024,4096\}$. To achieve a bounded latency for each processor, one common approach is the Cartesian partitioning, which can be obtained with hypergraph models (2D-b) [5], [7]. In this study, we follow a technique similar to the post-processing of Boman et al. [2] to manipulate an s2D partitioning in order to achieve a partitioning $(\mathrm{s} 2 \mathrm{D}-\mathrm{b})$ with bounded maximum latency. We will observe that the proposed method $s 2 \mathrm{D}-\mathrm{b}$ uniformly outperforms the state-of-the-art methods $2 \mathrm{D}-\mathrm{b}$ and $1 \mathrm{D}-\mathrm{b} \mathrm{b}$ in both the load balance and the total communicational volume. Finally, we compare the proposed heuristic for the s2D partitioning with the adapted version of the medium-grain partitioning Pelt and Bisseling [14], which is denoted with s2D-mg.

\section{A. Comparison with $1 D$ and $2 D$ methods}

Table I displays the properties of the matrices used to evaluate the performance of the proposed method S2D with respect to $1 \mathrm{D}$ and 2D methods, which refer to the 1D rowwise and the 2D fine-grain partitioning, respectively. We measure the performances with $K=\{16,64,256\}$ processors in terms of the partitioning qualities as well as their SpMV timings. We used a Cray XE6 system to measure the parallel performance. At each node, the system has two 16-core AMD processors with $2.3 \mathrm{GHz}$ clock frequency, and $32 \mathrm{~GB}$ memory. The interconnection network is a high speed 3D torus so called CRAY Gemini. We used one core at a node.

Table II displays a comparative summary of the performance results for $1 \mathrm{D}$ and $2 \mathrm{D}$ methods, and for the proposed method, s2D. For $1 \mathrm{D}$ and 2D methods, we used the column-net [3] and the row-column-net [5] hypergraph models to obtain the rowwise and the fine-grain partition, respectively. We used $\mathrm{PaToH}[4]$ as the hypergraph partitioner in default setting with imbalance tolerance of $3 \%$. For s2D, we used the heuristic presented in Section IV-B to build an s2D partition with respect to the vector partition induced by the rowwise partition that is used for 1D. Therefore, s2D and 1D exhibit the same communication pattern. As PaToH relies on randomization, we present the geometric averages of three runs for each partitioning instance.

We first investigate the performance in terms of the partitioning qualities. Here, we give all the numbers for $K=256$, though the statements are general. As seen in the table, s2D method reduces the total communication volume for each partitioning instance with respect to 1D. We observe that this reduction is correlated with the skewness of the nonzero distribution on rows. The matrices troheim and ASIC_680k exhibit very low and very high skewed nonzero distributions, and correspondingly, lead to $2 \%$ and $96 \%$ reductions in the communication volume, respectively. Also note that the computational load balance is either better than or equal to that of 1D. For instance, s2D reduces the load imbalance from $120 \%$ to $16 \%$, while improving the communication volume by $42 \%$ for $\mathrm{C}-\mathrm{b}$ ig. Notice that $2 \mathrm{D}$ method achieves a good computational balance $(0.1 \%$, on average), however the communication latency requirement is consistently larger than that of $1 \mathrm{D}$ and S2D methods, and displays approximately $60 \%$ increase in both total and maximum number of messages, on average. Also note that $\mathrm{S} 2 \mathrm{D}$ method exhibits communication volume comparable to $2 \mathrm{D}$, e.g., the average scaled volumes are 0.52 and 0.43 for s2D and 2D, respectively.

We now consider the parallel SpMV times. We display the speedup values with respect to a serial implementation. For six of the matrices, we observe the best speedup under s2D column, whereas on the matrices pkustk 12 and trdheim, 1D and 2D, respectively, exhibit the best performance (167.9 and 89.2 speedups, respectively). Moreover, s2D displays superior timing performance, on average, for all $K \in\{16,64,256\}$.

The table shows that the latency overhead of communication is a dominating factor (e.g., ASIC_680k achieves 2.4 and 9.2 speedups for 1D and S2D, respectively, on 256 processors) on the performance, especially when $K$ gets larger. In particular, the matrices with dense rows (e.g., c-big, ASIC_680k, and pattern1) tend to load a processor with very high number of messages to be received (or sent), in their parallel multiplication for 1D, and hence s2D. Notice that 2D method exhibit a similar performance for those matrices, since $2 \mathrm{D}$ implements the fine-grain partitioning method, which takes only the communication volume and the computational load balance into account. Thus, for sparse matrices with dense 


\begin{tabular}{|c|c|c|c|c|c|c|c|c|c|c|c|c|c|c|}
\hline \multirow{3}{*}{ name } & \multirow{3}{*}{$K$} & \multicolumn{5}{|c|}{$\begin{array}{l}\text { 1D partitioning } \\
\text { (1D) }\end{array}$} & \multicolumn{5}{|c|}{$\begin{array}{l}\text { 2D partitioning } \\
(2 \mathrm{D})\end{array}$} & \multicolumn{3}{|c|}{$\begin{array}{l}\text { s2D partitioning } \\
(\mathrm{s} 2 \mathrm{D})\end{array}$} \\
\hline & & \multirow{2}{*}{$\mathrm{LI} \%$} & \multicolumn{2}{|c|}{ Latency } & \multirow{2}{*}{$\lambda_{1 \mathrm{D}}$} & \multirow{2}{*}{$\mathrm{Sp}$} & \multirow{2}{*}{$\mathrm{LI} \%$} & \multicolumn{2}{|c|}{ Latency } & \multirow{2}{*}{$\frac{\lambda}{\lambda_{1 \mathrm{D}}}$} & \multirow{2}{*}{$\mathrm{Sp}$} & \multirow{2}{*}{$\mathrm{LI} \%$} & \multirow{2}{*}{$\frac{\lambda}{\lambda_{1 \mathrm{D}}}$} & \multirow{2}{*}{ Sp } \\
\hline & & & avg & $\max$ & & & & avg & $\max$ & & & & & \\
\hline \multirow{3}{*}{ crystk02 } & 16 & $1.0 \%$ & 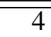 & 6 & $8.19 \mathrm{e} 3$ & 14.9 & $0.0 \%$ & 8 & 14 & 1.28 & 14.4 & $1.0 \%$ & 0.98 & 15.3 \\
\hline & 64 & $1.4 \%$ & 8 & 13 & $2.27 \mathrm{e} 4$ & 46.1 & $0.0 \%$ & 15 & 24 & 1.16 & 30.7 & $1.4 \%$ & 0.95 & 36.3 \\
\hline & 256 & $2.0 \%$ & 12 & 22 & $5.36 \mathrm{e} 4$ & 44.6 & $0.0 \%$ & 21 & 36 & 1.02 & 37.9 & $2.0 \%$ & 0.91 & 53.5 \\
\hline \multirow{3}{*}{ turon_m } & 16 & $0.4 \%$ & 5 & 7 & $1.76 \mathrm{e} 4$ & 15.5 & $1.4 \%$ & 8 & 11 & 0.48 & 15.8 & $0.3 \%$ & 0.80 & $\overline{15.4}$ \\
\hline & 64 & $0.6 \%$ & 6 & 8 & $3.89 \mathrm{e} 4$ & 43.4 & $1.2 \%$ & 9 & 15 & 0.49 & 44.2 & $0.6 \%$ & 0.81 & 39.1 \\
\hline & 256 & $0.9 \%$ & 8 & 14 & $7.92 \mathrm{e} 4$ & 88.3 & $1.1 \%$ & 13 & 21 & 0.52 & 117.2 & $0.9 \%$ & 0.84 & 128.0 \\
\hline \multirow{3}{*}{ trdheim } & 16 & $0.7 \%$ & 3 & 4 & $4.45 \mathrm{e} 3$ & 16.3 & $0.4 \%$ & 5 & 9 & 1.18 & 15.3 & $0.7 \%$ & 0.95 & 16.9 \\
\hline & 64 & $0.6 \%$ & 3 & 7 & $1.54 \mathrm{e} 4$ & 51.2 & $0.3 \%$ & 7 & 13 & 1.10 & 36.0 & $0.5 \%$ & 0.99 & 53.6 \\
\hline & 256 & $0.9 \%$ & 5 & 11 & $4.59 \mathrm{e} 4$ & 75.7 & $0.2 \%$ & 11 & 20 & 0.99 & 89.2 & $0.9 \%$ & 0.98 & 57.0 \\
\hline \multirow{3}{*}{ c-big } & 16 & $2.3 \%$ & 14 & 15 & $6.39 \mathrm{e} 4$ & 14.9 & $0.1 \%$ & 28 & 30 & 0.51 & $\overline{15.2}$ & $2.2 \%$ & 0.55 & 18.1 \\
\hline & 64 & $2.2 \%$ & 29 & 63 & $9.64 \mathrm{e} 4$ & 29.9 & $0.2 \%$ & 60 & 94 & 0.58 & 28.0 & $2.2 \%$ & 0.59 & 47.0 \\
\hline & 256 & $1.2^{*}$ & 34 & 253 & $1.38 \mathrm{e} 5$ & 6.9 & $0.2 \%$ & 64 & 285 & 0.62 & 9.3 & $16.6 \%$ & 0.58 & $\overline{7.7}$ \\
\hline \multirow{3}{*}{ 3dtube } & 16 & $0.9 \%$ & 5 & 9 & $1.73 \mathrm{e} 4$ & 16.5 & $0.0 \%$ & 10 & 14 & 1.03 & 15.9 & $0.8 \%$ & 0.92 & 16.6 \\
\hline & 64 & $0.8 \%$ & 6 & 17 & $3.88 \mathrm{e} 4$ & 62.1 & $0.0 \%$ & 12 & 21 & 1.17 & 49.6 & $0.8 \%$ & 0.98 & 60.9 \\
\hline & 256 & $12.9 \%$ & 8 & 36 & $9.32 \mathrm{e} 4$ & 100.6 & $0.0 \%$ & 17 & 48 & 1.13 & 74.7 & $12.9 \%$ & 0.80 & 123.0 \\
\hline \multirow{3}{*}{ ASIC_680k } & 16 & $1.4^{*}$ & 14 & 15 & $7.87 \mathrm{e} 5$ & 4.0 & $0.3 \%$ & 22 & 29 & 0.01 & 16.9 & $41.4 \%$ & 0.02 & $\overline{12.9}$ \\
\hline & 64 & $8.4^{*}$ & 24 & 57 & $7.95 \mathrm{e} 5$ & 3.7 & $0.3 \%$ & 35 & 89 & 0.01 & 35.8 & $41.7 \%$ & 0.02 & 37.0 \\
\hline & 256 & $36.7^{*}$ & 25 & 225 & $8.06 \mathrm{e} 5$ & 2.4 & $0.3 \%$ & 33 & 278 & 0.03 & 12.2 & $43.1 \%$ & 0.04 & $\overline{9.2}$ \\
\hline \multirow{3}{*}{ pkustk12 } & 16 & $0.9 \%$ & 4 & 14 & $4.57 \mathrm{e} 4$ & 16.7 & $0.6 \%$ & 13 & 22 & 0.30 & 16.3 & $0.9 \%$ & 0.93 & 16.7 \\
\hline & 64 & $1.2 \%$ & 9 & 25 & $1.39 \mathrm{e} 5$ & 64.7 & $0.2 \%$ & 20 & 58 & 0.24 & 53.8 & $1.2 \%$ & 0.91 & 63.5 \\
\hline & 256 & $8.3 \%$ & 18 & 40 & $2.39 \mathrm{e} 5$ & 167.9 & $0.2 \%$ & 22 & 149 & 0.32 & 45.7 & $8.3 \%$ & 0.55 & 162.9 \\
\hline \multirow{3}{*}{ pattern 1} & 16 & $2.2 \%$ & 12 & 15 & $6.07 \mathrm{e} 4$ & $\overline{20.2}$ & $0.2 \%$ & 30 & 30 & 0.39 & 18.8 & $2.0 \%$ & 0.69 & 20.8 \\
\hline & 64 & $2.2 \%$ & 23 & 63 & $1.14 \mathrm{e} 5$ & 55.4 & $0.4 \%$ & 54 & 106 & 0.51 & 63.6 & $2.2 \%$ & 0.70 & 66.8 \\
\hline & 256 & $2.2 \%$ & 42 & 252 & $2.79 \mathrm{e} 5$ & 23.2 & $0.5 \%$ & 63 & 308 & 0.41 & 23.9 & $2.2 \%$ & 0.85 & $\overline{23.1}$ \\
\hline \multirow{3}{*}{ geomean } & 16 & $1.9 \%$ & $\overline{6}$ & $\overline{10}$ & $3.34 \mathrm{e} 4$ & $\overline{13.7}$ & $0.1 \%$ & $\overline{13}$ & $\overline{18}$ & $\overline{0.36}$ & 16.0 & $1.5 \%$ & $\overline{0.51}$ & $\overline{16.4}$ \\
\hline & 64 & $2.6 \%$ & 10 & 23 & $7.09 \mathrm{e} 4$ & 35.5 & $0.1 \%$ & 20 & 39 & 0.40 & 41.2 & $1.8 \%$ & 0.54 & 49.2 \\
\hline & 256 & $10.6 \%$ & 15 & 54 & $1.38 \mathrm{e} 5$ & 34.4 & $0.1 \%$ & 25 & 85 & 0.43 & 37.2 & $4.8 \%$ & 0.52 & $\overline{43.5}$ \\
\hline
\end{tabular}

TABLE II: Performance results of S2D with respect to $1 \mathrm{D}$ and 2D, on $K \in\{16,64,256\}$ processors, in terms of the load imbalance (LI\%), where $x^{*}$ refers to $100 x \%$, the total communication volume ( $\lambda_{1 \mathrm{D}}$ is the exact volume for 1D, and the volume of $2 \mathrm{D}$ and $\mathrm{s} 2 \mathrm{D}$ are given as normalized to $\lambda_{1 \mathrm{D}}$ ), and the average and maximum number of messages sent by a processor. The communication pattern, and thus, the latency values of S2D are same with that of 1D. The speed-up values (Sp) are given with respect to serial implementation and the best of each rows is shown in bold, and the best of each matrix is underlined.

\begin{tabular}{|c|c|c|c|c|c|c|c|}
\hline \multirow{3}{*}{ name } & \multirow{3}{*}{\multicolumn{2}{|c|}{$\begin{array}{c}\text { Sp (best of } \\
1 \mathrm{D}, 2 \mathrm{D}, \mathrm{s} 2 \mathrm{D})\end{array}$}} & \multicolumn{5}{|c|}{$\begin{array}{l}\text { HP-based Cartesian partitioning } \\
(2 \mathrm{D}-\mathrm{b})\end{array}$} \\
\hline & & & \multirow{2}{*}{ LI\% } & \multicolumn{2}{|c|}{ Latency } & \multirow{2}{*}{$\frac{\lambda}{\lambda_{1 D}}$} & \multirow{2}{*}{$\mathrm{Sp}$} \\
\hline & & & & avg & $\max$ & & \\
\hline crystk02 & 53.5 & (s2D) & $2.7 \%$ & 13 & 19 & 1.18 & 62.2 \\
\hline turon_m & 128.0 & $(\mathbf{s} 2 \mathrm{D})$ & $3.3 \%$ & 9 & 16 & 1.24 & 79.5 \\
\hline trdheim & 89.2 & (2D) & $2.1 \%$ & 7 & 14 & 1.30 & 72.1 \\
\hline c-big & 9.3 & (2D) & $4.4 \%$ & 23 & 30 & 1.39 & 85.4 \\
\hline ASIC_680k & 12.2 & (2D) & $1.6^{*}$ & 21 & 29 & 1.24 & 37.9 \\
\hline 3dtube & 123.0 & (s2D) & $3.6 \%$ & 11 & 18 & 1.27 & 113.2 \\
\hline pkustk12 & 167.9 & (1D) & $3.7 \%$ & 15 & 26 & 1.16 & 186.3 \\
\hline pattern1 & 23.9 & (2D) & $14.0 \%$ & 24 & 30 & 0.91 & 214.0 \\
\hline geomean & 43.5 & $(\mathrm{~s} 2 \mathrm{D})$ & $6.3 \%$ & 14 & 22 & 1.20 & 92.2 \\
\hline
\end{tabular}

TABLE III: Comparison of Cartesian partitioning that is based on hypergraph partitioning $(2 \mathrm{D}-\mathrm{b})$ with the best of $1 \mathrm{D}, 2 \mathrm{D}$, and S2D methods for $K=256$ in terms of speed-up (Sp) with respect to the serial implementation. The communication volume is given as normalized to that of 1D. The load imbalance (LI\%) of ASIC_680k, i.e., 1.6*, refers to $\sim 160 \%$.

rows, partitionings that bound the maximum latency are essential to achieve efficient parallelization.

Table III compares the previously discussed methods 1D, 2D, and s2D, with Cartesian partitioning 2D-b, called checkerboard [5], [7]. As seen in the table, in five of the test cases, $2 \mathrm{D}-\mathrm{b}$ demonstrates better speedups than all $1 \mathrm{D}$,
2D, and s2D. Notice that the improvement is much more pronounced for those matrices with dense rows. For instance, 2D-b improves speedup from 12.2 (for 2D) to 37.9 despite its large load imbalance of $160 \%$.

\section{B. Comparison with recent work}

1) Cartesian methods: Table IV shows the properties of the matrices that we used to assess the proposed s2D-b method for $K \in\{256,1024,4096\}$. Two of the matrices (c-big and ASIC_680k) exist also in the previous setup. Another two are obtained through SNAP [13], where com-Youtube is the online social network of Youtube, and rmat_20 is a R-MAT [8] graph (edges made undirected) with parameters $a=0.57, b=c=0.19$, and $d=0.05$. The matrices in this set have some dense rows (see $d_{\max }$ in the table), e.g., the maximum number of nonzeros of a row of $\mathrm{c}-\mathrm{big}$ is approximately $6 \%$ and $1 \%$ of the total number of columns and nonzeros, respectively, and ins 2 contains a row that is full of nonzeros.

We propose $s 2 \mathrm{D}-\mathrm{b}$ in order to bound the maximum latency requirement at the expense of extra communication phase, similar to that of Boman et al. [2]. Unlike the standard Cartesian partitionings, s2D-b enables that the input-vector entries and the partial output-vector results are communicated together at each communication phase. We first compare 


\begin{tabular}{l|rrrr|l} 
name & $n$ & $n n z$ & $d_{\mathrm{avg}}$ & $d_{\max }$ & description \\
\hline boyd2 & 466316 & 1500397 & 3.2 & 93263 & optimization \\
lp1 & 534388 & 1643420 & 3.1 & 249644 & optimization \\
c-big & 345241 & 2340859 & 6.8 & 19579 & non-linear opt. \\
ASIC_680k & 682862 & 2638997 & 3.9 & 388489 & optimization \\
ins2 & 309412 & 2751484 & 8.9 & 309413 & circuit sim. \\
com-Youtube & 1157827 & 5975248 & 5.2 & 28755 & Youtube social \\
rajat30 & 643994 & 6175244 & 9.6 & 454747 & circuit sim. \\
rmat_20 & 1048576 & 8174570 & 7.8 & 23716 & Graph500 ben. \\
\hline
\end{tabular}

TABLE IV: Properties of the test matrices with dense rows to be used in discussion of the proposed $\mathrm{s} 2 \mathrm{D}-\mathrm{b}$ method. The sparse matrices are displayed in increasing order of their nonzero counts $(n n z)$. Here, $d_{\text {avg }}$ and $d_{\max }$ refer to the average and maximum nonzero counts of rows, respectively.

s2D-b with S2D in order to show the interplay between the latency and the bandwidth requirements. Table $\mathrm{V}$ displays the performance of $1 \mathrm{D}, \mathrm{s} 2 \mathrm{D}$ and $\mathrm{s} 2 \mathrm{D}-\mathrm{b}$ in the set of matrices with dense rows, where 1D is also included in order to provide a unified perspective. As seen in the table, 1D method fails to achieve an acceptable partitioning quality, in terms of either the computational load, or the communication latency, or bandwidth. The maximum number of nonzeros of a row is $1.07 e 5$, and total number of nonzeros is $3.26 e 6$. Thus, it is theoretically impossible to achieve perfect balance for larger than 30 processors with 1D methods, on average, and the balance degenerates linearly with the increasing number of processors. Owing to the increased flexibility of nonzeros in partitioning, s2D method exhibist reasonable performance on the computational load. For instance, we observe $83.8 \%$ load imbalance, on average for $K=4096$ of s2D, which is equivalent to performing $\sim 2229$ processors with perfect balance. As s2D-b employs the same nonzero partition with s2D, we observe such improvement on the computational load balance for $\mathrm{s} 2 \mathrm{D}-\mathrm{b}$, as well.

Table $\mathrm{V}$ displays a significant reduction in the total communication volume with S2D when compared to 1D methods. For instance, on average, we observe a reduction of $95 \%$ and $80 \%$, for $K=256$ and 4096 , respectively. As seen in the table, s2D-b increases communication volume with respect to s2D. Nevertheless, the communication volume of s2D-b is still significantly less than 1D, e.g., $94 \%$ and $76 \%$ reduction, for $K=256$ and 4096, respectively, on average.

In terms of the communication latency, 1D, and equivalently s2D, perform in such a way that the maximum number of messages sent by a processor is in the order of $K$, e.g., 3579 messages for $K=4096$, on average. By the virtue of the block layout of processors and the two-phase communication of $\mathrm{s} 2 \mathrm{D}-\mathrm{b}$, we observe that the maximum latency requirement is in the order of $\sqrt{K}$, e.g., 90 messages for $K=4096$, on average, for $\mathrm{s} 2 \mathrm{D}-\mathrm{b}$.

Table VI compares S2D-b with the state-of-the-art methods 2D-b [7] and 1D-b [2]. For s2D-b, we use the same partitions with the ones displayed in Table V. Moreover, we construct $1 \mathrm{D}-\mathrm{b}$ on the same vector partition with that of s2D-b. All methods exhibit comparable performance for both maximum and average latency requirement. For instance, the total and the maximum number of messages is $19 \times K$ and 90 for $2 \mathrm{D}-\mathrm{b}$ (cf. $18 \times K$ and 90 for $\mathrm{s} 2 \mathrm{D}-\mathrm{b}$, and equivalently for 1D-b), respectively, with $K=4096$, on average. Therefore, we compare the methods only in terms of the computational load balance and the total communication volume.

We first discuss the computational load balance. As seen in Table VI, 2D-b displays $75.1 \%$ load imbalance, and we observe that the imbalance increases linearly with $K$, e.g., $202.8 \%$ and $508.2 \%$ for $K=1024$ and $K=4096$, respectively, on average. The load balance is much more dramatic for 1D-b. For instance, we observe $841.1 \%$ load imbalance with 4096 processors, on average, for 1D-b. The proposed method $\mathrm{s} 2 \mathrm{D}-\mathrm{b}$, on the other hand, distributes the computational load in considerably more balance than $2 \mathrm{D}-\mathrm{b}$ and $1 \mathrm{D}-\mathrm{b}$, e.g., 83.8\% load imbalance with 4096 processors, on average.

In terms of the total communication volume, s2D-b is the clear winner for this set of matrices, where $2 \mathrm{D}-\mathrm{b}$ and 1D-b perform comparable to each other. The high bandwidth requirement of $2 \mathrm{D}-\mathrm{b}$ and $1 \mathrm{D}-\mathrm{b}$ methods is inevitable due to the high degree rows. The method $\mathrm{s} 2 \mathrm{D}-\mathrm{b}$ alleviates this problem by sending the precomputed partial $\mathbf{y}$-vector results along with the $\mathrm{x}$-vector entries. As seen in the table, s2D-b performs uniformly better than $2 \mathrm{D}-\mathrm{b}$ and $1 \mathrm{D}-\mathrm{b}$ methods for real-life matrices, and, on average, displays $84 \%$ reduction in the communication volume compared to $2 \mathrm{D}-\mathrm{b}$ for $K=4096$.

As a result, we observe that on the matrices with high degree rows/columns, the proposed method $s 2 \mathrm{D}-\mathrm{b}$ improves the state-of-the-art methods $1 \mathrm{D}-\mathrm{b}$ and $2 \mathrm{D}-\mathrm{b}$ in both the total communication volume and the computational load balance.

2) Medium-grain partitioning: Table VII compares the proposed method s2D with s2D-mg Recall that s2D-mg refers to the medium-grain method [14] adapted to produce an s2D partition. As seen in the table, s2D-mg achieves better load balance than s2D. For instance, for $K=256$ on average, s2D achieves $52.3 \%$ load imbalance, whereas s2D-mg displays a load imbalance of $4.8 \%$. On the positive side, s2D exhibits significantly less communication volume than s2D-mg, for instance, it nearly halves the bandwidth requirement for $K=256$, on average. However, for some instances, s2D-mg has better performance, such as ASIC_680k and rajat 30 . Nevertheless, having large number of processors is in favor of s2D. For instance, we observe s2D reduces the communication volume by $16 \%$ for ASIC_680k when $K=4096$, with respect to $s 2 \mathrm{D}-\mathrm{mg}$.

As seen in the table, the total latency requirement of s2D is considerably less than that of s2D-mg in general. On average, $\mathrm{s} 2 \mathrm{D}-\mathrm{mg}$ and $\mathrm{s} 2 \mathrm{D}$ require $38 \times K$ and $30 \times K$ messages to be communicated, respectively, for $K=4096$. There are cases where the improvement in the total latency requirement is much more pronounced, such as boyd2, where the total number of messages for s2D-mg and s2D are $33 \times K$ and $6 \times K$, respectively, for $K=4096$.

To compare, s2D reduces the communication volume significantly, and exhibits better latency performance, whereas s2D-mg achieves better load balance. We notice that the performance gap closes with increasing $K$ for each criterium. 


\begin{tabular}{|c|c|c|c|c|c|c|c|c|c|c|}
\hline \multirow{3}{*}{ name } & \multirow{3}{*}{$K$} & \multicolumn{4}{|c|}{$1 D$} & \multicolumn{2}{|c|}{$S 2 D$} & \multicolumn{3}{|c|}{$s 2 D-b$} \\
\hline & & \multirow{2}{*}{$\mathrm{LI} \%$} & \multicolumn{2}{|c|}{ Latency } & \multirow{2}{*}{$\lambda_{1 \mathrm{D}}$} & \multirow{2}{*}{$\mathrm{LI} \%$} & \multirow{2}{*}{$\frac{\lambda}{\lambda_{1 \mathrm{D}}}$} & \multicolumn{2}{|c|}{ Latency } & \multirow{2}{*}{$\frac{\lambda}{\lambda_{1 D}}$} \\
\hline & & & avg & $\max$ & & & & avg & $\max$ & \\
\hline \multirow{3}{*}{ boyd 2} & 256 & $14.9^{*}$ & $\overline{5}$ & 239 & $2.30 \mathrm{e} 5$ & $33.1 \%$ & 0.01 & $\overline{\overline{4}}$ & $\overline{25}$ & $\overline{0.01}$ \\
\hline & 1024 & $62.7^{*}$ & 5 & 945 & $2.40 \mathrm{e} 5$ & $32.3 \%$ & 0.02 & 5 & 38 & 0.02 \\
\hline & 4096 & $253.6^{*}$ & 6 & 3731 & $2.51 \mathrm{e} 5$ & $29.8 \%$ & 0.09 & 5 & 66 & 0.10 \\
\hline \multirow{3}{*}{ lp1 } & 256 & $41.6^{*}$ & 2 & 225 & $2.50 \mathrm{e} 5$ & $38.3 \%$ & 0.00 & 2 & 16 & 0.00 \\
\hline & 1024 & $169.4^{*}$ & 2 & 918 & $2.51 \mathrm{e} 5$ & $63.7 \%$ & 0.01 & 2 & 32 & 0.01 \\
\hline & 4096 & $680.5^{*}$ & 2 & 3984 & $2.57 \mathrm{e} 5$ & $1.3^{*}$ & 0.04 & 2 & 64 & 0.04 \\
\hline \multirow{3}{*}{ c-big } & 256 & $2.1^{*}$ & 34 & 253 & $1.38 \mathrm{e} 5$ & $66.1 \%$ & 0.58 & 21 & 30 & 0.82 \\
\hline & 1024 & $11.2^{*}$ & 24 & 1005 & $1.96 \mathrm{e} 5$ & $1.2^{*}$ & 0.62 & 23 & 57 & 0.86 \\
\hline & 4096 & $47.8^{*}$ & 15 & 3563 & $3.16 \mathrm{e} 5$ & $1.4^{*}$ & 0.64 & 17 & 107 & 0.84 \\
\hline \multirow{3}{*}{ ASIC_680k } & 256 & $41.5^{*}$ & 25 & 225 & $8.06 \mathrm{e} 5$ & $61.4 \%$ & 0.03 & 18 & 28 & 0.05 \\
\hline & 1024 & $168.9^{*}$ & 21 & 897 & $8.26 \mathrm{e} 5$ & $63.4 \%$ & 0.06 & 19 & 46 & 0.08 \\
\hline & 4096 & $678.8^{*}$ & 15 & 3578 & $8.63 \mathrm{e} 5$ & $65.9 \%$ & 0.10 & 15 & 78 & 0.12 \\
\hline \multirow{3}{*}{ ins 2} & 256 & $29.0^{*}$ & 24 & 218 & $6.91 \mathrm{e} 5$ & $45.0 \%$ & 0.01 & 14 & 29 & 0.01 \\
\hline & 1024 & $119.1^{*}$ & 22 & 839 & $7.00 \mathrm{e} 5$ & $54.3 \%$ & 0.03 & 14 & 56 & 0.04 \\
\hline & 4096 & $479.2^{*}$ & 21 & 3310 & $7.75 \mathrm{e} 5$ & $61.4 \%$ & 0.11 & 13 & 101 & 0.12 \\
\hline \multirow{3}{*}{ com-Youtube } & 256 & $1.7^{*}$ & 232 & 255 & $1.60 \mathrm{e} 6$ & $2.4^{*}$ & 0.65 & 30 & 30 & 0.91 \\
\hline & 1024 & $9.7^{*}$ & 468 & 964 & $2.20 \mathrm{e} 6$ & $2.9^{*}$ & 0.69 & 60 & 62 & 0.98 \\
\hline & 4096 & $41.8^{*}$ & 366 & 3306 & $2.83 \mathrm{e} 6$ & $3.3^{*}$ & 0.73 & 104 & 126 & 1.05 \\
\hline \multirow{3}{*}{ rajat30 } & 256 & $17.9^{*}$ & 18 & 212 & $1.20 \mathrm{e} 6$ & $28.7 \%$ & 0.05 & 14 & 28 & 0.06 \\
\hline & 1024 & $74.4^{*}$ & 20 & 823 & $1.31 \mathrm{e} 6$ & $34.5 \%$ & 0.08 & 18 & 50 & 0.10 \\
\hline & 4096 & $300.6^{*}$ & 20 & 3237 & $1.48 \mathrm{e} 6$ & $42.0 \%$ & 0.14 & 19 & 78 & 0.16 \\
\hline \multirow{3}{*}{ rmat_20 } & 256 & $1.4 \%$ & 255 & 255 & $4.53 \mathrm{e} 6$ & $34.5 \%$ & 0.72 & 30 & 30 & 0.94 \\
\hline & 1024 & $3.1^{*}$ & 1019 & 1022 & $5.94 \mathrm{e} 6$ & $82.0 \%$ & 0.81 & 62 & 62 & 1.09 \\
\hline & 4096 & $15.3^{*}$ & 1337 & 4011 & $6.88 \mathrm{e} 6$ & $76.0 \%$ & 0.90 & 124 & 126 & 1.27 \\
\hline \multirow{3}{*}{ geomean } & 256 & $5.3^{*}$ & 26 & 235 & $6.65 \mathrm{e} 5$ & $52.3 \%$ & 0.05 & 12 & 27 & 0.06 \\
\hline & 1024 & $38.9^{*}$ & 32 & 924 & $7.65 \mathrm{e} 5$ & $71.7 \%$ & 0.10 & 16 & 49 & 0.12 \\
\hline & 4096 & $163.7^{*}$ & 30 & 3579 & $8.90 \mathrm{e} 5$ & $83.8 \%$ & 0.20 & 18 & 90 & 0.24 \\
\hline
\end{tabular}

TABLE V: Performance results of s2D-b with respect to 1D and s2D for $K \in\{256,1024,4096\}$ in terms of the load imbalance (LI\%, with $x^{*}$ being $100 x \%$ ), the total communication volume ( $\lambda_{1 \mathrm{D}}$ is that of $1 \mathrm{D}$, and those of s2D and S2D-b are given as normalized to $\lambda_{1 \mathrm{D}}$ ), and the average and maximum number of messages sent by a processor. The communication pattern and the latency values of S2D and $1 \mathrm{D}$ are the same. The load imbalance values of s2D and s2D-b are the same.

\section{CONCLUSION AND RESEARCH DIRECTIONS}

We introduced semi-two-dimensional (s2D) partitioning of a sparse matrix for parallel sparse matrix vector multiply (SpMV) operations. This partitioning has more flexibility than one dimensional (rowwise or columnwise) partitioning schemes. Yet, there is a constraint which makes it less flexible than nonzero-based, two dimensional partitionings. Each nonzero is assigned to the processor that has the associated input- or output-vector entry. We modified the organization of the standard SpMV operation to take advantage of this constraint to confine all communication operations in a single phase. That is, s2D has a data distribution more general than $1 \mathrm{D}$ and less general than 2D, yet it enables a single phase of communication in SpMV as 1D schemes.

We proposed a two-step method to obtain an s2D partitioning. In the first step, a given input- and output-vector partition is used to impose a block structure in the input matrix. In the second step, horizontal blocks of nonzeros are carefully re-assigned to processors to optimize the total communication volume with respect to the partition imported in the first step, or traded off with the load balance. Using a suit of reallife matrices, we observed speed-ups with respect to serial execution with s2D method (49.2) compared to 1D rowwise (35.5) and 2D fine-grain (41.2), on average, using a Cray
XE6 system. We also observed that the merit of the proposed method is much more evident in matrices having rows (or columns) with a high number of nonzeros. For those matrices, we saw $97 \%$ to $85 \%$ improvement on the communication volume for 256 and 4096 processors, respectively, on average.

We achieved significant improvement on the communication volume, but the load balance was not as good as that of finegrain (or medium-grain [14]) partitionings. This depends on the vector partition assumed in the first step of the proposed heuristic. More sophisticated heuristics that also take square and vertical blocks of off-diagonal blocks into account can be considered in the second step to mitigate this dependency. More advanced methods to find input-vector, output-vector, and nonzero partition, simultaneously can also be devised.

By making use of an idea from a recent work [2], we devised a variant ( $s 2 D-b)$ of the proposed $s 2 \mathrm{D}$ method, which bounds the maximum latency requirement by a number in the order of square root of the number of processors. According to our experiments, $\mathrm{s} 2 \mathrm{D}-\mathrm{b}$ outperformed the best alternatives (which have the same latency bound) in both load balance (e.g., from $508.2 \%$ to $83.8 \%$ with 4096 processors) and the communication volume (e.g., $82 \%$ reduction for 4096 processors). However, s2D-b requires more elaboration, particularly in terms of SpMV timings, which we leave as future work. 


\begin{tabular}{|c|c|c|c|c|c|c|c|}
\hline \multirow[b]{2}{*}{ name } & \multirow[b]{2}{*}{$K$} & \multicolumn{2}{|c|}{$2 \mathrm{D}-\mathrm{b}$} & \multicolumn{2}{|c|}{$1 \mathrm{D}-\mathrm{b}[2]$} & \multicolumn{2}{|c|}{$s 2 D-b$} \\
\hline & & $\mathrm{LI} \%$ & $\lambda_{2 \mathrm{D}-\mathrm{b}}$ & $\mathrm{LI} \%$ & $\frac{\lambda}{\lambda_{2 D-b}}$ & $\mathrm{LI} \%$ & $\frac{\lambda}{\lambda_{2 D-b}}$ \\
\hline \multirow{3}{*}{ boyd2 } & 256 & $54.2 \%$ & $4.25 \mathrm{e} 5$ & $1.8^{*}$ & 0.96 & $33.1 \%$ & 0.00 \\
\hline & 1024 & $2.0^{*}$ & $4.43 \mathrm{e} 5$ & $5.3^{*}$ & 0.98 & $32.3 \%$ & 0.01 \\
\hline & 4096 & $4.8^{*}$ & $4.55 \mathrm{e} 5$ & $13.5^{*}$ & 0.99 & $29.8 \%$ & 0.05 \\
\hline \multirow{3}{*}{ lp1 } & 256 & $2.5^{*}$ & $4.98 \mathrm{e} 5$ & $3.3^{*}$ & 0.97 & $38.3 \%$ & 0.00 \\
\hline & 1024 & $6.2^{*}$ & $5.00 \mathrm{e} 5$ & $6.7^{*}$ & 0.98 & $63.7 \%$ & 0.00 \\
\hline & 4096 & $16.2^{*}$ & $5.04 \mathrm{e} 5$ & $13.6^{*}$ & 0.99 & $1.3^{*}$ & 0.02 \\
\hline \multirow{3}{*}{ c-big } & 256 & $48.7 \%$ & $1.92 \mathrm{e} 5$ & $84.5 \%$ & 1.01 & $66.1 \%$ & 0.59 \\
\hline & 1024 & $64.1 \%$ & $2.72 \mathrm{e} 5$ & $1.1^{*}$ & 0.98 & $1.2^{*}$ & 0.62 \\
\hline & 4096 & $2.8^{*}$ & $4.15 \mathrm{e} 5$ & $3.1^{*}$ & 0.97 & $1.4^{*}$ & 0.64 \\
\hline \multirow{3}{*}{ ASIC_680k } & 256 & $2.0^{*}$ & $1.00 \mathrm{e} 6$ & $1.7^{*}$ & 0.99 & $61.4 \%$ & 0.04 \\
\hline & 1024 & $5.0^{*}$ & $1.03 \mathrm{e} 6$ & $4.4^{*}$ & 0.92 & $63.4 \%$ & 0.06 \\
\hline & 4096 & $11.7^{*}$ & $1.07 \mathrm{e} 6$ & $9.9^{*}$ & 0.92 & $65.9 \%$ & 0.10 \\
\hline \multirow{3}{*}{ ins2 } & 256 & $1.8^{*}$ & $1.27 \mathrm{e} 6$ & $2.9^{*}$ & 0.94 & $45.0 \%$ & 0.01 \\
\hline & 1024 & $4.8^{*}$ & $1.34 \mathrm{e} 6$ & $7.1^{*}$ & 0.87 & $54.3 \%$ & 0.02 \\
\hline & 4096 & $10.3^{*}$ & $1.40 \mathrm{e} 6$ & $19.3^{*}$ & 0.79 & $61.4 \%$ & 0.07 \\
\hline \multirow{3}{*}{ com-Youtube } & 256 & $1.2^{*}$ & $2.99 \mathrm{e} 6$ & $2.4^{*}$ & 0.57 & $2.4^{*}$ & 0.48 \\
\hline & 1024 & $1.3^{*}$ & $3.72 \mathrm{e} 6$ & $3.6^{*}$ & 0.64 & $2.9^{*}$ & 0.58 \\
\hline & 4096 & $4.8^{*}$ & $4.51 \mathrm{e} 6$ & $13.7^{*}$ & 0.71 & $3.3^{*}$ & 0.66 \\
\hline \multirow{3}{*}{ rajat30 } & 256 & $1.0^{*}$ & $2.24 \mathrm{e} 6$ & $3.3^{*}$ & 0.81 & $28.7 \%$ & 0.03 \\
\hline & 1024 & $2.8^{*}$ & $2.41 \mathrm{e} 6$ & $6.8^{*}$ & 0.82 & $34.5 \%$ & 0.05 \\
\hline & 4096 & $7.4^{*}$ & $2.58 \mathrm{e} 6$ & $15.9^{*}$ & 0.82 & $42.0 \%$ & 0.09 \\
\hline \multirow{3}{*}{ rmat_20 } & 256 & $3.7 \%$ & $3.59 \mathrm{e} 6$ & $3.7 \%$ & 0.87 & $34.5 \%$ & 1.19 \\
\hline & 1024 & $41.3 \%$ & $4.97 \mathrm{e} 6$ & $51.5 \%$ & 0.91 & $82.0 \%$ & 1.31 \\
\hline & 4096 & $47.8 \%$ & $6.51 \mathrm{e} 6$ & $1.1^{*}$ & 0.94 & $76.0 \%$ & 1.34 \\
\hline \multirow{3}{*}{ geomean } & 256 & $75.1 \%$ & $1.03 \mathrm{e} 6$ & $1.3^{*}$ & 0.88 & $52.3 \%$ & 0.04 \\
\hline & 1024 & $2.0^{*}$ & $1.18 \mathrm{e} 6$ & $3.3^{*}$ & 0.88 & $71.7 \%$ & 0.08 \\
\hline & 4096 & $5.1^{*}$ & $1.35 \mathrm{e} 6$ & $8.4^{*}$ & 0.89 & $83.8 \%$ & 0.16 \\
\hline
\end{tabular}

TABLE VI: Performance results of $s 2 \mathrm{D}-\mathrm{b}$ with respect to 2D-b and 1D-b [2] for $K \in\{256,1024,4096\}$ in terms of the load imbalance (LI\%, with $x^{*}$ being $100 x \%$ ), and the communication volume $\left(\lambda_{2 D-b}\right.$ is that of $2 \mathrm{D}-\mathrm{b}$; those of $1 \mathrm{D}-\mathrm{b}$ and $\mathrm{s} 2 \mathrm{D}-\mathrm{b}$ are given as normalized to $\lambda_{2 \mathrm{D}-\mathrm{b}}$ ).

\begin{tabular}{l|r|rrr|rrr}
\multirow{2}{*}{ name } & \multirow{2}{*}{$K$} & \multicolumn{3}{c|}{ s2D-mg [14] } & \multicolumn{3}{c}{ s2D } \\
\cline { 3 - 8 } & & LI\% & Lat. & $\lambda_{\text {mg }}$ & LI\% & Lat. & $\frac{\lambda}{\lambda_{\text {mg }}}$ \\
\hline \hline \multirow{3}{*}{ boyd2 } & 256 & $0.1 \%$ & 105 & $1.25 \mathrm{e} 5$ & $33.1 \%$ & 5 & 0.01 \\
& 1024 & $0.4 \%$ & 85 & $1.29 \mathrm{e} 5$ & $32.3 \%$ & 5 & 0.04 \\
& 4096 & $1.0 \%$ & 33 & $1.48 \mathrm{e} 5$ & $29.8 \%$ & 6 & 0.16 \\
\hline \multirow{3}{*}{ lp1 } & 256 & $9.6 \%$ & 2 & $5.10 \mathrm{e} 2$ & $38.3 \%$ & 2 & 0.91 \\
& 1024 & $9.8 \%$ & 2 & $2.15 \mathrm{e} 3$ & $63.7 \%$ & 2 & 0.90 \\
& 4096 & $10.8 \%$ & 2 & $1.06 \mathrm{e} 4$ & $1.3 *$ & 2 & 1.01 \\
\hline \multirow{3}{*}{ c-big } & 256 & $43.2 \%$ & 40 & $8.82 \mathrm{e} 4$ & $66.1 \%$ & 34 & 0.90 \\
& 1024 & $43.5 \%$ & 31 & $1.39 \mathrm{e} 5$ & $1.2^{*}$ & 24 & 0.88 \\
& 4096 & $47.5 \%$ & 20 & $2.33 \mathrm{e} 5$ & $1.4^{*}$ & 15 & 0.87 \\
\hline \multirow{3}{*}{ ASIC_680k } & 256 & $13.1 \%$ & 28 & $2.08 \mathrm{e} 4$ & $61.4 \%$ & 25 & 1.34 \\
& 1024 & $13.2 \%$ & 23 & $4.27 \mathrm{e} 4$ & $63.4 \%$ & 21 & 1.07 \\
& 4096 & $13.3 \%$ & 15 & $1.02 \mathrm{e} 5$ & $65.9 \%$ & 15 & 0.84 \\
\hline \multirow{3}{*}{ ins2 } & 256 & $5.0 \%$ & 19 & $1.41 \mathrm{e} 4$ & $45.0 \%$ & 24 & 0.53 \\
& 1024 & $5.1 \%$ & 25 & $3.84 \mathrm{e} 4$ & $54.3 \%$ & 22 & 0.62 \\
& 4096 & $5.7 \%$ & 18 & $1.01 \mathrm{e} 5$ & $61.4 \%$ & 21 & 0.87 \\
\hline \multirow{3}{*}{ com-Youtube } & 256 & $1.2 *$ & 243 & $1.49 \mathrm{e} 6$ & $2.4^{*}$ & 232 & 0.69 \\
& 1024 & $1.2 *$ & 552 & $2.00 \mathrm{e} 6$ & $2.9^{*}$ & 468 & 0.76 \\
& 4096 & $1.3 *$ & 411 & $2.54 \mathrm{e} 6$ & $3.3 *$ & 366 & 0.82 \\
\hline \multirow{2}{*}{ rajat30 } & 256 & $0.8 \%$ & 20 & $4.15 \mathrm{e} 4$ & $28.7 \%$ & 18 & 1.31 \\
& 1024 & $1.1 \%$ & 24 & $9.09 \mathrm{e} 4$ & $34.5 \%$ & 20 & 1.21 \\
& 4096 & $1.3 \%$ & 23 & $1.98 \mathrm{e} 5$ & $42.0 \%$ & 20 & 1.07 \\
\hline \multirow{2}{*}{ rmat_20 } & 256 & $1.0 \%$ & 255 & $3.27 \mathrm{e} 6$ & $34.5 \%$ & 255 & 0.99 \\
& 1024 & $39.5 \%$ & 972 & $4.86 \mathrm{e} 6$ & $82.0 \%$ & 1019 & 0.99 \\
& 4096 & $60.3 \%$ & 1072 & $6.14 \mathrm{e} 6$ & $76.0 \%$ & 1337 & 1.01 \\
\hline \multirow{2}{*}{ geomean } & 256 & $4.8 \%$ & 39 & $6.54 \mathrm{e} 4$ & $52.3 \%$ & 26 & 0.52 \\
& 1024 & $9.4 \%$ & 50 & $1.24 \mathrm{e} 5$ & $71.7 \%$ & 32 & 0.61 \\
& 4096 & $11.9 \%$ & 38 & $2.42 \mathrm{e} 5$ & $83.8 \%$ & 30 & 0.74 \\
\hline \multirow{2}{*}{} & & & & & & &
\end{tabular}

TABLE VII: Performance results of S2D with respect to s2D-mg [14] for $K \in\{256,1024,4096\}$ in terms of the load imbalance (LI\%, with $x^{*}$ being $100 x \%$ ), the average number of messages (Lat.), and the total communication volume ( $\lambda_{\mathrm{mg}}$ is that of $\mathrm{s} 2 \mathrm{D}-\mathrm{mg}$; that of $\mathrm{s} 2 \mathrm{D}$ is given as normalized to $\lambda_{\mathrm{mg}}$ ).

\section{ACKNOWLEDGEMENTS}

Bora Uçar is supported by ANR project SOLHAR (ANR13- MONU-0007).

\section{REFERENCES}

[1] R. H. Bisseling, Parallel Scientific Computation: A Structured Approach using BSP and MPI. Oxford University Press, Oxford, UK, 2004.

[2] E. G. Boman, K. D. Devine, and S. Rajamanickam, "Scalable matrix computations on large scale-free graphs using 2D graph partitioning," in Proc. International Conference on High Performance Computing, Networking, Storage and Analysis. ACM, 2013, pp. 50:1-50:12.

[3] Ü. V. Çatalyürek and C. Aykanat, "Hypergraph-partitioning-based decomposition for parallel sparse-matrix vector multiplication," IEEE T. Parall. Distr., vol. 10, no. 7, pp. 673-693, 1999.

[4] _- PaToH: A Multilevel Hypergraph Partitioning Tool, Version 3.2, Bilkent Univ., Dept. Computer Engineering, Ankara, 06533 Turkey. Available at http://bmi.os u.edu/ umit/software.htm, 1999.

[5] Ü. V. Çatalyürek, C. Aykanat, and B. Uçar, "On two-dimensional sparse matrix partitioning: Models, methods, and a recipe," SIAM J. Sci. Comput., vol. 32, no. 2, pp. 656-683, 2010.

[6] Ü. V. Çatalyürek and C. Aykanat, "A fine-grain hypergraph model for 2D decomposition of sparse matrices," in Proc. 15th IPDPS, San Francisco, CA, 2001, p. 118.

[7] Ü. V. Çatalyürek and C. Aykanat, "A hypergraph-partitioning approach for coarse-grain decomposition," in Proc. Supercomputing, ACM/IEEE 2001 Conference, Denver, Colorado, 2001, p. 42.

[8] D. Chakrabarti, Y. Zhan, and C. Faloutsos, "R-mat: A recursive model for graph mining," in Proc. SDM, 2004.

[9] T. A. Davis and Y. Hu, "The University of Florida sparse matrix collection," ACM Trans. Math. Softw., vol. 38, no. 1, pp. 1:1-1:25, 2011.

[10] A. L. Dulmage and N. S. Mendelsohn, "Coverings of Bipartite Graphs," Canadian Journal of Mathematics, no. 10, pp. 517-534, 1958.

[11] K. Kaya, B. Uçar, and U. V. Çatalyürek, "Analysis of partitioning models and metrics in parallel sparse matrix-vector multiplication," in Proc. PPAM, Warsaw, Poland, 2014, pp. 174-184.

[12] V. Kuhlemann and P. S. Vassilevski, "Improving the communication pattern in matrix-vector operations for large scale-free graphs by disaggregation," SIAM J. Sci. Comput., vol. 35, no. 5, pp. S465-S486, 2013.

[13] J. Leskovec and R. Sosič, "SNAP: A general purpose network analysis and graph mining library in C++," http://snap.stanford.edu/snap, 2014.

[14] D. M. Pelt and R. H. Bisseling, "A medium-grain method for fast 2D bipartitioning of sparse matrices," in Proc. 28th IPDPS, Phoenix, Arizona, May 2014, pp. 529-539.

[15] A. Pothen and C.-J. Fan, "Computing the block triangular form of a sparse matrix," ACM Trans. Math. Softw., vol. 16, no. 4, pp. 303-324, 1990.

[16] R. O. Selvitopi, M. Ozdal, and C. Aykanat, "A novel method for scaling iterative solvers: Avoiding latency overhead of parallel sparse-matrix vector multiplies," IEEE T. Parall. Distr., vol. PP, no. 99, pp. 1-1, 2014.

[17] B. Uçar and C. Aykanat, "Revisiting hypergraph models for sparse matrix partitioning," SIAM Rev., vol. 49, no. 4, pp. 595-603, 2007.

[18] B. Vastenhouw and R. H. Bisseling, "A two-dimensional data distribution method for parallel sparse matrix-vector multiplication," SIAM Rev., vol. 47, no. 1, pp. 67-95, 2005.

[19] R. S. Xin, J. E. Gonzalez, M. J. Franklin, and I. Stoica, "Graphx: A resilient distributed graph system on spark," in First International Workshop on Graph Data Management Experiences and Systems, ser. GRADES '13. New York, NY, USA: ACM, 2013, pp. 2:1-2:6.

[20] A. Yoo, A. H. Baker, R. Pearce, and V. E. Henson, "A scalable eigensolver for large scale-free graphs using 2D graph partitioning," in Proc. International Conference for High Performance Computing, Networking, Storage and Analysis. ACM, 2011, pp. 63:1-63:11.

[21] A. Yoo, E. Chow, K. Henderson, W. McLendon, B. Hendrickson, and U. V. Çatalyürek, "A scalable distributed parallel breadth-first search algorithm on BlueGene/L," in Proc. 2005 ACM/IEEE Conference on Supercomputing. Washington, DC, USA: IEEE Computer Society, 2005, p. 25. 